\title{
A 3G/WiFi-enabled 6LoWPAN-based u-healthcare system for ubiquitous real-time monitoring and data logging
}

\begin{abstract}
Ubiquitous healthcare (U-healthcare) systems are expected to offer flexible and resilient high-end technological solutions enabling remote monitoring of patients health status in realtime and provisioning of feedback and remote actions by healthcare providers. In this paper, we present a 6LowPAN based U-healthcare platform that contributes to the realization of the above expectation. The proposed system comprises two sensor nodes sending temperature data and ECG signals to a remote processing unit. These sensors are being assigned an IPv6 address to enable the Internet-of-Things (IoT) functionality. A 6LowPAN-enabled edge router, connected to a PC, is serving as a base station through a serial interface, to collect data from the sensor nodes. Furthermore, a program interfacing through a Serial-Line-InternetProtocol (SLIP) and running on the PC provides a network interface that receives IPv6 packets from the edge router. The above system is enhanced by having the application save readings from the sensors into a file that can be downloaded by a remote server using a free Cloud service such as UbuntuOne. This enhancement makes the system robust against data loss especially for outdoor healthcare services, where the $3 \mathrm{G} / 4 \mathrm{G}$ connectivity may get lost because of signal quality fluctuations. The system provided a proof of concept of successful remote U-healthcare monitoring illustrating the IoT functionality and involving $3 \mathrm{G} / 4 \mathrm{G}$ connectivity while being enhanced by a cloud-based backup.
\end{abstract}

